\section{Haemorrhagic ulcerative duodenitis in a patient with COVID-19 infection: clinical improvement following treatment with budesonide}

To cite: Awwad I, Greuel S, Tacke F, et al. Haemorrhagic ulcerative duodenitis in a patient with COVID-19 infection: clinical improvement following treatment with budesonide. BMJ Open Gastro 2021;8:e000757. doi:10.1136/ bmjgast-2021-000757

Received 1 August 2021 Accepted 6 October 2021
D Check for updates

(C) Author(s) (or their employer(s)) 2021. Re-use permitted under CC BY-NC. No commercial re-use. See rights and permissions. Published by BMJ.

${ }^{1}$ Department of Gastroenterology and Hepatology, Charité Universitätsmedizin Berlin, Berlin, Germany ${ }^{2}$ Department of Pathology, Charité Universitätsmedizin Berlin, Berlin, Germany

Correspondence to Dr Ibrahim Awwad; ibrahim.awwad@charite.de

Dr Christian Fischer; christian.fischer@charite.de

\section{ABSTRACT}

We present a case of a male patient in his mid-30s with COVID-19-induced lung failure requiring extracorporeal membrane oxygenation, who needed an emergency oesophagogastroduodenoscopy due to major upper gastrointestinal bleeding. Endoscopy exposed severe ulcerative duodenitis with diffuse mucosal bleeding. While CT angiography did not show any signs of ischaemia, histopathology revealed duodenitis with substantial inflammatory cell infiltrates consisting of neutrophils and $\mathrm{CD}^{+} \mathrm{T}$ lymphocytes with equal $\mathrm{CD}^{+} / \mathrm{CD}^{+}$distribution. Since the composition of cell infiltrates coincides with changes in inflammatory patterns of the respiratory mucosa from patients with COVID-19 and in COVID-19associated enterocolitis, and systemic dexamethasone treatment became standard of care in ventilated intensive care unit patients with COVID-19 infection, we initiated an individualised therapeutic attempt to treat the duodenitis with topical enteral budesonide. Followup oesophagogastroduodenoscopies within 4 weeks of enteral budesonide administration revealed a full clinical and histological healing of the duodenal mucosa with marked reduction of neutrophilic and lymphocytic infiltrates.

To our knowledge, the current report is the first description of enteral budesonide treatment of duodenitis in a patient with COVID-19 infection and warrants further investigation, whether budesonide might constitute a novel therapeutic strategy for the management of COVID-19related intestinal mucosal damage.

\section{CASE REPORT}

A male patient in his mid-30s with no history of pre-existing illness was admitted to the intensive care unit (ICU) with a COVID-19 infection confirmed by a positive SARSCoV-2 PCR test in the nasopharyngeal swab. Four weeks following admission, the patient required extracorporeal membrane oxygenation (ECMO) due to acute respiratory failure.

Another 4 weeks later, while being on ECMO therapy, an upper gastrointestinal bleeding occurred with tarry stools and drop in haemoglobin levels, which required on average up to two transfusions of packed red cells every 24 hours. The initial oesophagogastroduodenoscopy (EGD) revealed a severe haemorrhagic ulcerative duodenitis encompassing the entire circumference of the duodenum (figure 1A,B), while the mucosal surface of the stomach and the oesophagus appeared unaltered. There were signs of diffuse duodenal bleeding, originating from the inflamed and contact-vulnerable duodenal mucosa. However, there was no indication of bleeding from distinct vascular stumps requiring immediate endoscopic haemostasis. Notably, CT angiography did not reveal any causal evidence for mucosal ischaemia. While heparin-based anticoagulation was necessary due to ECMO therapy (partial thromboplastin time: 40-45 s), vasopressor therapy was performed at low-dose norepinephrine only $(0.04 \mu \mathrm{g} / \mathrm{kg} / \mathrm{min})$.

By histopathology, biopsies from the duodenal mucosa depicted both active and chronic duodenitis, reflected by an abundant neutrophilic inflammation as well as lymphocellular infiltrates, mainly consisting of $\mathrm{CD}^{+} \mathrm{T}$ lymphocytes. The numbers of $\mathrm{CD}^{+}$and $\mathrm{CD}^{+} \mathrm{T}$ lymphocytes were equally distributed, whereas $\mathrm{CD}^{+} 0^{+} \mathrm{B}$ lymphocytes were only sporadically present. Furthermore, an increased epithelial regeneration and mucin depletion were observed (figure 1C), while haemorrhages and extensive mucosal necrosis were absent. The capillaries of the lamina propria were devoid of inflammation (as a surrogate for endothelialitis) and without evidence of intravascular coagulation or fibrin microthrombi. At this time point, SARS-CoV-2 PCR tests taken from the duodenal biopsies turned out negative.

We decided to initiate an individualised therapeutic attempt to treat the severe duodenitis with topical budesonide. The choice of topical enteral budesonide was based on the 


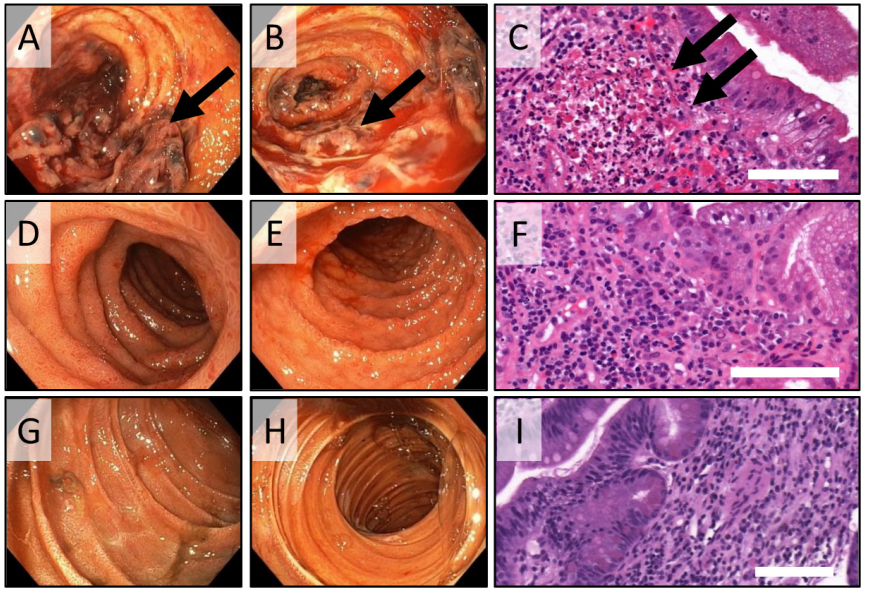

Figure 1 Endoscopic and histopathological characteristics of a patient with COVID-19 suffering from severe ulcerative and haemorrhagic duodenitis before treatment $(A-C)$, as well as following 2 weeks (D-F) and 4 weeks $(G-I)$ of treatment with topical enteral budesonide, respectively. $(A, B)$ Pictures illustrate endoscopic findings with severe haemorrhagic ulcerative duodenitis (indicated by arrow in A) encompassing the entire circumference of the duodenum. (C) H\&E stain of biopsies from the duodenum depicts mucosal inflammation with neutrophilic and lymphocytic infiltrates (indicated by arrows) as well as necrosis. (D-F) Follow-up endoscopy after 2 weeks illustrates macroscopic improvement of duodenal mucosal damage $(D, E)$ with a moderate reduction of the inflammatory neutrophilic and lymphocytic cell infiltrates in biopsies of duodenal mucosa (F). (G-I) following 4 weeks of enteral budesonide treatment endoscopy showed a complete healing of the duodenal mucosa $(\mathrm{G}, \mathrm{H})$, and corresponding histopathology revealed a pronounced decrease of the neutrophilic and lymphocytic cell infiltrates (I). Scale bars correspond to $100 \mu \mathrm{m}$.

consideration that systemic dexamethasone treatment became the standard of care in ventilated ICU patients with severe COVID-19 infection, ${ }^{1}$ and the composition of the cell infiltrates was consistent with inflammatory patterns of the respiratory mucosa in patients with COVID-19 ${ }^{2}$ and in COVID-19-associated enterocolitis. ${ }^{3}$ Budesonide was administered enterally at a dose of $3 \mathrm{mg}$ three times per day with a sodium chloride solution via nasogastric tube.

Two weeks after the start of budesonide treatment, a follow-up EGD was performed. On endoscopy, duodenitis was clearly improved with less ulceration, whereas the corresponding histopathological analysis from duodenal biopsies depicted only a slight improvement of the cellular inflammation (figure 1D-F). In particular, the abundance of neutrophils and inflammatory T-lymphocytic cell infiltrates only moderately decreased as compared with the previous findings (figure $1 \mathrm{C}, \mathrm{F}$ ).

Hence, we repeated the EGD 4 weeks following the start of therapy with enteral budesonide and were ultimately able to demonstrate a relevant healing of the duodenitis (figure 1G-I), both macroscopically and histologically with a pronounced reduction of the neutrophilic and lymphocellular infiltrates as compared with the previous histopathological analyses (figure 1C,F,I). Clinically, mucosal healing was accompanied by stabilisation of haemoglobin levels, cessation of gastrointestinal bleeding and red blood cell transfusion need.

Although there is an increasing body of reports and series published on COVID-19-related gastrointestinal bleeding, the exact underlying mechanisms have not been elucidated yet. ${ }^{4-7}$ Interestingly, ACE 2, which has been identified as the cellular receptor for SARS$\mathrm{CoV}-2,{ }^{289}$ is abundantly expressed in the glandular cells of gastric, duodenal and rectal epithelia, ${ }^{9}$ indicating the entry of SARS-CoV-2 into the host cells of the gastrointestinal tract. In a recent clinical and laboratory review, only 6 out of 95 patients with COVID-19 infection ultimately needed to undergo an EGD due to gastrointestinal symptoms, with only one case of these 6 patients suffering from ulcer bleeding in the oesophagus. ${ }^{5}$ Notably, SARS-CoV-2 RNA transcript expression was inconsistent and could be detected in biopsies from duodenal mucosa in only three out of these six patients who underwent an EGD. ${ }^{5}$

A most recent report evaluated clinicopathological findings in a series of 22 patients with severe COVID-19 disease, undergoing bowel resection due to acute ischaemic enterocolitis $^{3}$ and revealed that COVID-19-associated ischaemic enterocolitis typically involved small vessel fibrin thrombi and perivascular neutrophils. ${ }^{3}$ In sharp contrast, capillaries in the duodenal biopsies in our case were without any signs of fibrin microthrombi, but histology rather revealed abundant neutrophilic inflammation and lymphocellular infiltrates. While this composition of neutrophilic and lymphocellular infiltrates is not specific for COVID-19 infection, it is yet compatible with reported histopathological changes in the cellular inflammatory patterns of the respiratory mucosa from patients with severe COVID-19 infection. ${ }^{2}$ Most strikingly, in our case, histology depicted an extensive neutrophilic inflammation of the duodenal mucosa, and ultimate mucosal healing was furthermore associated with a major decrease in neutrophilic infiltrates on treatment with enteral budesonide. The role of neutrophils in COVID-19 disease is still poorly understood, but recent evidence has emerged that SARS-CoV-2-triggered immune responses involve tissue recruitment of neutrophils to the lungs. ${ }^{10}$ Furthermore, neutrophilic activity compulsory contributes to and intensifies the severity of COVID-19 mediated lung damage by degranulation of proinflammatory cytokines and release of neutrophil extracellular traps, ${ }^{11} 12$ even in the absence of detectable viral SARS-CoV-2 expression in the target tissue. ${ }^{13}$ While SARSCoV-2-mediated neutrophil function in the gastrointestinal tract has not been addressed yet, it is tempting to speculate that gastrointestinal mucosa damage in COVID-19 disease may be ascribed to similar pathophysiological mechanisms of neutrophilic inflammation as reported in COVID-19 lung damage. ${ }^{12}$

Our data therefore suggest that our current case of severe ulcerative duodenitis might be related to systemic COVID-19 infection, although RNA transcript expression for SARS-CoV-2 in biopsy specimens from the duodenal 
mucosa was absent. This notion is furthermore supported by the fact that macrovascular ischaemia could be repeatedly ruled out by CT-angiography, although the absence of mesenteric artery occlusion in CT scans does not exclude microvascular ischaemia. ${ }^{14}$ However, our histological analyses of duodenal mucosa biopsies did not reveal histopathological features, which are essential characteristics for mucosal ischaemia, such as haemorrhage and extensive mucosal necrosis. ${ }^{15}$ Moreover, vasopressor therapy was at low-dose norepinephrine only, and hence unlikely a risk factor for intestinal (microvascular) ischaemia.

Emerging evidence indicates that small vessel fibrin thrombi and perivascular neutrophilic infiltrates represent features of COVID-19-associated enterocolitis. ${ }^{3}$ Although we could not detect fibrin thrombi in microvessels of the lamina propria, the neutrophilic infiltrates in the duodenal mucosa in our patient are compatible with reported histopathological features of COVID-19-associated cellular inflammatory patterns of the intestinal mucosa and other organs, such as the respiratory mucosa. ${ }^{2}$ The absence of fibrin microthrombi in our case might be due to the fact, that tissue biopsies only reach vessels in the lamina propria, and hence fibrin microthrombi of the submucosal layer could have been missed. Moreover, it remains a matter of debate whether anticoagulation (due to ECMO therapy) could have prevented the formation of microthrombi. On the other side, the use of anticoagulation or antiplatelet agents per se was not a risk factor for gastrointestinal bleeding in a large cohort of hospitalised patients with COVID-19. ${ }^{7}$

Finally, disseminated stress ulcers due to critical illness conceivably represent another cause of diffuse bleeding from damaged duodenal mucosa but might be less important, since intravenous proton pump inhibitor therapy was continuously administered in standard-dose on admission to the ICU and even increased to high dose, when the upper gastrointestinal bleeding had started.

Based on the outcome of the UK RECOVERY study, systemic dexamethasone treatment has been found to reduce mortality rate and hence became the standard of care in ventilated ICU patients with severe COVID-19 infection. ${ }^{1}$ To date, clinical data on the use of systemic steroids regarding gastrointestinal symptoms in patients with COVID-19 is lacking, while the efficacy of topical budesonide in the clinical management of patients with COVID-19 infection has become available only for the treatment of prolonged anosmia. ${ }^{16}$

To our knowledge, the presented case report is the first description of topical enteral budesonide treatment of haemorrhagic ulcerative duodenitis in a patient with severe COVID-19 infection. Despite the severe alteration of the tissue with pronounced neutrophilic inflammation, we observed a full clinical and histological recovery on oral administration of budesonide. Although this case does not allow to conclude that budesonide treatment was solely responsible for the rapid recovery, our case report warrants further investigations, whether topical budesonide might constitute a new therapeutic strategy for the management of COVID-19-related gastrointestinal symptoms, such as severe mucosal damage and bleeding.

Contributors Study concept and design: IA; data acquisition: IA, CF and SG; data interpretation: IA, CF, SG and FT; manuscript preparation and editing: IA and CF; manuscript review: IA, CF and FT.

Funding The authors have not declared a specific grant for this research from any funding agency in the public, commercial or not-for-profit sectors.

Competing interests None declared.

Patient consent for publication Not applicable.

Provenance and peer review Not commissioned; externally peer reviewed.

Data availability statement All data relevant to the study are included in the article or uploaded as supplementary information. n/a.

Open access This is an open access article distributed in accordance with the Creative Commons Attribution Non Commercial (CC BY-NC 4.0) license, which permits others to distribute, remix, adapt, build upon this work non-commercially, and license their derivative works on different terms, provided the original work is properly cited, appropriate credit is given, any changes made indicated, and the use is non-commercial. See: http://creativecommons.org/licenses/by-nc/4.0/.

ORCID iDs

Ibrahim Awwad http://orcid.org/0000-0002-9686-4000

Frank Tacke http://orcid.org/0000-0001-6206-0226

\section{REFERENCES}

1 RECOVERY Collaborative Group, Horby P, Lim WS, et al. Dexamethasone in hospitalized patients with Covid-19. N Engl J Med 2021;384:693-704.

2 Deshmukh V, Motwani R, Kumar A, et al. Histopathological observations in COVID-19: a systematic review. J Clin Pathol 2021;74:76-83.

3 Zhang ML, Jacobsen F, Pepe-Mooney BJ. Clinicopathological findings in patients with COVID-19-associated ischaemic enterocolitis. Histopathology 2021;118.

4 Amarapurkar AD, Vichare P, Pandya N, et al. Haemorrhagic enteritis and COVID-19: causality or coincidence. J Clin Pathol 2020;73:686.

5 Lin L, Jiang X, Zhang Z, et al. Gastrointestinal symptoms of 95 cases with SARS-CoV-2 infection. Gut 2020;69:997-1001.

6 Martin TA, Wan DW, Hajifathalian K, et al. Gastrointestinal bleeding in patients with coronavirus disease 2019: a matched case-control study. Am J Gastroenterol 2020;115:1609-16.

7 Trindade AJ, Izard S, Coppa K, et al. Gastrointestinal bleeding in hospitalized COVID-19 patients: a propensity score matched cohort study. J Intern Med 2021;289:887-94.

8 Rezaei M, Ziai SA, Fakhri S, et al. Ace2: its potential role and regulation in severe acute respiratory syndrome and COVID-19. J Cell Physiol 2021;236:2430-42.

9 Xiao F, Tang M, Zheng X, et al. Evidence for gastrointestinal infection of SARS-CoV-2. Gastroenterology 2020;158:1831-3.

10 Hemmat N, Derakhshani A, Bannazadeh Baghi H, et al. Neutrophils, crucial, or harmful immune cells involved in coronavirus infection: a bioinformatics study. Front Genet 2020;11:641.

11 Veras FP, Pontelli MC, Silva CM, et al. SARS-CoV-2-triggered neutrophil extracellular traps mediate COVID-19 pathology. J Exp Med 2020;217. doi:10.1084/jem.20201129. [Epub ahead of print: 07 Dec 2020].

12 Cavalcante-Silva LHA, Carvalho DCM, Lima Éssia de Almeida, et al. Neutrophils and COVID-19: the road so far. Int Immunopharmacol 2021;90:107233.

13 Stahl K, Bräsen JH, Hoeper MM, et al. Absence of SARS-CoV-2 RNA in COVID-19-associated intestinal endothelialitis. Intensive Care Med 2021;47:359-60.

14 Korswagen L, Voerman HJ, Peterse JL. Ischemic duodenitis without involvement of the large abdominal arteries. Endoscopy 2007;39 Suppl 1:E271.

15 Uberti G, Goldblum JR, Allende DS. Ischemic enterocolitis and its differential diagnosis. Semin Diagn Pathol 2014;31:152-64.

16 Daval M, Corré A, Palpacuer C, et al. Efficacy of local budesonide therapy in the management of persistent hyposmia in COVID-19 patients without signs of severity: a structured summary of a study protocol for a randomised controlled trial. Trials 2020;21:666. 